\title{
Adjuvant therapy of endometrial cancer: "taxane or not taxane, this is the question"
}

\author{
Vito Lorusso, Simona Vallarelli, Francesco Giotta \\ Medical Oncology Unit, IRCCS Istituto Tumori “Giovanni Paolo II”, Bari, Italy \\ Correspondence to: Vito Lorusso. Chief of Medical Oncology Unit, IRCCS Istituto Tumori “Giovanni Paolo II”, Viale Orazio Flacco, 65, 70124 Bari, \\ Italy. Email: vitolorusso@me.com. \\ Comment on: Nomura H, Aoki D, Michimae H, et al. Effect of Taxane Plus Platinum Regimens vs Doxorubicin Plus Cisplatin as Adjuvant \\ Chemotherapy for Endometrial Cancer at a High Risk of Progression: A Randomized Clinical Trial. JAMA Oncol 2019;5:833-40.
}

Submitted Apr 24, 2019. Accepted for publication May 06, 2019.

doi: $10.21037 /$ tcr.2019.05.03

View this article at: http://dx.doi.org/10.21037/tcr.2019.05.03

Endometrial cancer is the fifth cause of cancer in women, with an incidence rate of more than 300,000 new cases per year worldwide (1). In USA it is the most common gynecologic malignancy, with approximately 61,880 newly diagnosed cases and 12,160 deaths expected in 2019 (2). Most cases are diagnosed at an early stage and have relatively good survival rates; however, women diagnosed with recurrent disease or advanced stages have a very poor prognosis (3). The standard treatment of endometrial carcinoma is hysterectomy plus bilateral salpingooophorectomy. After surgical treatment, patients with low risk early stage receive only adjuvant radiation, whereas chemotherapy +/- radiotherapy is given to high risk early stages, locally advanced disease not amenable of surgery, or to those patients with residual disease. Almost $75 \%$ of endometrial cancers are diagnosed at an early stage (FIGO stage I or II): for these patients the 5 -year overall survival ranges from $74 \%$ to $91 \%$; for patients with FIGO stage III, the 5 -year overall survival is $57-66 \%$, whereas for stage IV disease is only $20-26 \%$. Lymph node metastatic involvement is a critical prognostic factor for disease free survival. In fact, for those patients without lymph node metastasis, 5-year disease free survival is about $90 \%, 60-70 \%$ in patients with involvement of pelvic lymph nodes, and $30-40 \%$ for those with paraaortic lymph node metastasis, respectively $(4,5)$. In patients with advanced endometrial cancer, chemotherapy is still the mainstay of treatment, because targeted therapy as well as immunotherapy, have not yet demonstrated any utility. The standard chemotherapy for advanced disease is the combination of cisplatin plus doxorubicin that demonstrated improved response rates and PFS (5.7 vs.
3.8 months) compared with doxorubicin alone in a phase 3 study, although OS was not increased (9.0 vs. 9.2 months) (6). In order to compare radiotherapy to chemotherapy, the GOG-122 study randomized 396 patients with stage III or IV (including patients with maximum of $2 \mathrm{~cm}$ of postoperative residual disease) to whole-abdominal radiotherapy or chemotherapy with doxorubicincisplatin (7). This study demonstrated, that patients in the chemotherapy arm had a significantly improved 5 -year survival rate $(55 \%$ vs. $42 \%$, respectively), although they suffered of greater toxicity. To further improve these results, the GOG-177 study compared the combination of three drugs (doxorubicin, cisplatin and paclitaxel) with filgrastim support (TAP) with standard doxorubicin and cisplatin. This study demonstrated a significantly improved response rate (57\% vs. $34 \%$, respectively), PFS (8.3 vs. 5.3 months, respectively), and OS (15.3 vs. 12.3 months, respectively; $\mathrm{P}=0.037$ ) as compared to standard, but also significantly higher neurotoxicity for the triple drug combination (8). Following this study, GOG-209 compared carboplatin and paclitaxel to the TAP regimen with the aim to develop a less toxic regimen. The findings of this study were that the combination of carboplatin plus paclitaxel was not inferior to TAP in terms of PFS (14 months in both arms) and OS (32 vs. 38 months, respectively; HR 1.01) (9). On the other hand, the toxicity profile for the two-drug combination was significantly more favorable as compared to the triple combination, and this regimen became a new standard for chemotherapy trials.

In earlier stages it is very difficult to compare the results of "adjuvant chemotherapy" of the various studies 
because of the different eligible criteria adopted. In fact, the characteristics of patients affected with endometrial carcinoma submitted to surgery plus/minus radiotherapy included in "adjuvant chemotherapy" phase 3 trials, range from high risk stage I, through stage IIIC with positive lymph node metastases (10-13). In fact, an Italian study (10) and the JGOG2033 (11) included patients with stage IC with myometrial invasion exceeding half through stage III. In a study of 2 randomized clinical trials (12) one trial (NSGOEC-9501/EORTC-55991) enrolled patients who had cancer in stages I, II, IIIA with positive cytology, and stage IIIC with positive pelvic lymph node metastasis, while the other trial (MaNGO ILIADE-III) enrolled patients who had cancer in stage IIB through III (excluding stage III with positive cytology). In the GOG122 study, patients had stage III cancer with less than $2 \mathrm{~cm}$ residual tumor and stage IV cancer (7). The present study (14) encompassed the patient population investigated in previous phase 3 studies of adjuvant therapy rendering the scenario still more confusing. In fact, the eligibility criteria of the Nomura et al. study included: high risk stages I, II, III and IV patients who had tumor not extending beyond the abdominal cavity and had $2 \mathrm{~cm}$ or greater residual tumor. Moreover, the results of this study are also flawed by the huge percent of the patients who did not complete the planned six cycles of therapy, ranging from $17.1 \%$ to $24 \%$ in the three arms.

Nomura et al. in the conclusions claim that taxane plus platinum regimens did not demonstrate a survival benefit over treatment with doxorubicin plus cisplatin, however, in the subgroup analysis they observed an improved PFS in patients with lymph node metastases treated with docetaxel plus cisplatin or paclitaxel plus carboplatin as compared to those treated with the "standard" doxorubicin plus cisplatin. Thus, at the end of the story the superiority of taxane containing regimens over doxorubicin/cisplatin was not demonstrated, albeit the toxicity, in particular cardiac toxicity, is certainly inferior for taxane regimens which are actually widely used also in adjuvant setting, based on the extrapolation of results achieved in advanced stages studies.

\section{Acknowledgments}

Funding: None.

\section{Footnote}

Provenance and Peer Review: This article was commissioned and reviewed by the Section Editor Hengwei Liu, MD,
$\mathrm{PhD}$ (Department of Obstetrics and Gynecology, Union Hospital, Tongji Medical College, Huazhong University of Science and Technology, Wuhan, China).

Conflicts of Interest: The authors have completed the ICMJE uniform disclosure form (available at http://dx.doi. org/10.21037/tcr.2019.05.03). The authors have no conflicts of interest to declare.

Ethical Statement: The authors are accountable for all aspects of the work in ensuring that questions related to the accuracy or integrity of any part of the work are appropriately investigated and resolved.

Open Access Statement: This is an Open Access article distributed in accordance with the Creative Commons Attribution-NonCommercial-NoDerivs 4.0 International License (CC BY-NC-ND 4.0), which permits the noncommercial replication and distribution of the article with the strict proviso that no changes or edits are made and the original work is properly cited (including links to both the formal publication through the relevant DOI and the license). See: https://creativecommons.org/licenses/by-nc-nd/4.0/.

\section{References}

1. IARC 2013. The International Agency for Research on Cancer. GLOBOCAN 2012: Estimated cancer incidence, mortality and prevalence worldwide in 2012. Available online: http://globocan.iarc.fr/Pages/fact_sheets_ population.aspx

2. American Cancer Society: Key Statistics for Endometrial Cancer, 2019.

3. SEER Stat Fact Sheets: endometrial Cancer. 2019; accessed April 23, 2019.

4. Zaino RJ, Kurman RJ, Diana KL, et al. Pathologic models to predict outcome for women with endometrial adenocarcinoma: the importance of the distinction between surgical stage and clinical stage--a Gynecologic Oncology Group study. Cancer 1996;77:1115-21.

5. Hirai M, Hirono M, Oosaki T, et al. Prognostic factors relating to survival in uterine endometrioid carcinoma. Int J Gynaecol Obstet 1999;66:155-62.

6. Thigpen JT, Brady MF, Homesley HD, et al. Phase III trial of doxorubicin with or without cisplatin in advanced endometrial carcinoma: a gynecologic oncology group study. J Clin Oncol 2004;22:3902-8.

7. Randall ME, Filiaci VL, Muss H, et al. Randomized phase 
III trial of whole-abdominal irradiation versus doxorubicin and cisplatin chemotherapy in advanced endometrial carcinoma: a Gynecologic Oncology Group Study. J Clin Oncol 2006;24:36-44.

8. Fleming GF, Brunetto VL, Cella D, et al. Phase III trial of doxorubicin plus cisplatin with or without paclitaxel plus filgrastim in advanced endometrial carcinoma: a Gynecologic Oncology Group Study. J Clin Oncol 2004;22:2159-66.

9. Miller DS, Filiaci G, Mannel R, et al. Randomized Phase III Noninferiority Trial of First Line Chemotherapy for Metastatic or Recurrent Endometrial Carcinoma: A Gynecologic Oncology Group Study. Gynecol Oncol 2012;125:771-3

10. Maggi R, Lissoni A, Spina F, et al. Adjuvant chemotherapy vs radiotherapy in high-risk endometrial carcinoma: results of a randomised trial. Br J Cancer 2006;95:266-71.

11. Susumu N, Sagae S, Udagawa Y, et al. Randomized phase

Cite this article as: Lorusso V, Vallarelli S, Giotta F. Adjuvant therapy of endometrial cancer: "taxane or not taxane, this is the question”. Transl Cancer Res 2019;8(6):2220-2222. doi: 10.21037/ tcr.2019.05.03
III trial of pelvic radiotherapy versus cisplatin-based combined chemotherapy in patients with intermediateand high-risk endometrial cancer: a Japanese Gynecologic Oncology Group study. Gynecol Oncol 2008;108:226-33.

12. Hogberg T, Signorelli M, de Oliveira CF, et al. Sequential adjuvant chemotherapy and radiotherapy in endometrial cancer--results from two randomised studies. Eur J Cancer 2010;46:2422-31.

13. Homesley HD, Filiaci V, Gibbons SK, et al. A randomized phase III trial in advanced endometrial carcinoma of surgery and volume directed radiation followed by cisplatin and doxorubicin with or without paclitaxel: A Gynecologic Oncology Group study. Gynecol Oncol 2009;112:543-52.

14. Nomura H, Aoki D, Michimae H, et al. Effect of Taxane Plus Platinum Regimens vs Doxorubicin Plus Cisplatin as Adjuvant Chemotherapy for Endometrial Cancer at a High Risk of Progression: A Randomized Clinical Trial. JAMA Oncol 2019;5:833-40. 